\title{
Tópicos Emergentes em Insuficiência Cardíaca: Nova Era do Tratamento Farmacológico
}

\author{
Emerging Topics in Heart Failure: New Era of Pharmacological Treatment
}

Fabiana G. Marcondes-Braga, ${ }^{1}$ Felix J. A. Ramires, ${ }^{1,2}$ Estêvão Lanna Figueiredo, ${ }^{3}$ J José Albuquerque Figueiredo

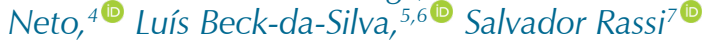

Instituto do Coração do Hospital das Clínicas da Faculdade de Medicina da Universidade de São Paulo (InCor.HCFMUSP), ${ }^{1}$ São Paulo, SP - Brasil Hospital do Coração (HCOR), ${ }^{2}$ São Paulo, SP - Brasil

Instituto Orizonti e Hospital Vera Cruz, ${ }^{3}$ Belo Horizonte, MG - Brasil

Universidade Federal do Maranhão (UFMA), ${ }^{4}$ São Luís, MA - Brasil

Hospital de Clínicas de Porto Alegre, ${ }^{5}$ Porto Alegre, RS - Brasil

Universidade Federal do Rio Grande do Sul, ${ }^{6}$ Porto Alegre, RS - Brasil

Hospital das Clínicas da Universidade Federal de Goiás, ${ }^{7}$ Goiânia, GO - Brasil

Carta científica referente ao Heart Failure Summit Brazil 2020 / Departamento de Insuficiência Cardíaca - DEIC/SBC

\section{Introdução}

Nas últimas décadas, avanços no tratamento farmacológico e no uso de dispositivos implantáveis trouxeram mudanças no prognóstico de pacientes com insuficiência cardíaca de fração de ejeção reduzida (ICFEr). No entanto, ainda existe risco residual e alta morbimortalidade. Novas medicações, com ação em diferentes mecanismos fisiopatológicos, complementam a ação sobre o sistema neuro-humoral e remodelamento e seus benefícios ocorrem em adição à terapia padrão otimizada. A tabela 1 descreve os principais estudos sobre o tratamento medicamentoso da IC.

\section{Terapia Padrão Otimizada}

Sistema Renina-angiotensina-aldosterona (IECAs/ BRAs/ AMRs)

Estudos de desfechos clínicos importantes, como mortalidade e hospitalização, evidenciaram a fundamental importância do sistema renina-angiotensina-aldosterona (SRAA), com a atenuação da ação da angiotensina (AngII), utilizando os Inibidores da enzima de conversão da angiotensinal (IECAs) ou os bloqueadores do receptor da Angll (BRAs), sendo esses últimos indicados em intolerantes aos IECAs.

Além da atenuação da ação da Angll, os antagonistas mineralocorticoides (ARMs) também se mostraram fundamentais na modulação do SRAA, tanto em pacientes mais sintomáticos (CF III-IV, NYHA) quanto naqueles com menos sintomas (CFII).

\section{Palavras-chave}

Insuficiência Cardíaca; Tratamento Farmacológico, Insuficiência Cardíaca de Fração de Ejeção Reduzida.

\section{Correspondência: Salvador Rassi •}

Rua T-36, 3229 apto 300. CEP 74223-055, Setor Bueno, Goiânia, GO - Brasil E-mail: srassi@cardiol.br

Artigo recebido em 14/10/2020, revisado em 14/10/2020,

aceito em 14/10/2020

DOI: https://doi.org/10.36660/abc.20201106
Inibição da Neprilisina (associada ao bloqueio do receptor de AngII)

Mais recentemente, a atenuação da ação deletéria da Angll, associada ao efeito protetor dos peptídeos natriuréticos, utilizando uma nova classe de fármacos, os inibidores da neprilisina e dos receptores da AngII (INRAs), cuja molécula atualmente disponível é o sacubitril/valsartana, mostrou-se superior aos IECAs, tanto na redução de mortalidade quanto de hospitalização por IC (HIC). Inicialmente, era indicada em substituição aos IECAs/BRAs apenas em pacientes ambulatoriais que se mantivessem sintomáticos (CFII-III, NYHA). Novos dados sustentam a possibilidade de início de tratamento com sacubitril/valsartana, ao invés de IECAs/BRAs, em pacientes com IC nova, assim como em pacientes ainda internados por IC descompensada após estabilização.

\section{Bloqueio do Sistema Nervoso Simpático}

Apesar dos avanços terapêuticos recentes, os $\beta$-bloqueadores (carvedilol, succinato de metoprolol e bisoprolol) continuam fundamentais no tratamento da ICFEr, por comprovadamente reduzirem sintomas, morte (por todas as causas, por IC e súbita) e hospitalizações em pacientes sintomáticos ou com disfunção ventricular assintomática. ${ }^{8-10}$ Devem ser iniciados em todos os pacientes, associados aos bloqueadores do SRAA, em doses reduzidas e tituladas àquelas utilizadas nos ensaios clínicos.

Terapias farmacológicas adicionais

\section{Nitrato/Hidralazina}

A associação de nitrato e de hidralazina demonstrou ser efetiva na redução de desfechos sólidos, mortalidade total e HIC em pacientes autodeclarados negros. A associação pode ainda ser indicada para pacientes que apresentam piora da função renal e/ou hipercalemia em uso de IECA/BRA/INRA.

\section{Ivabradina}

A ivabradina inibe seletivamente a corrente If no tecido do nó sinusal, reduzindo a frequência cardíaca (FC), que é 


\section{Carta Científica}

\begin{tabular}{|c|c|c|c|c|}
\hline Estudos & & População & Desfecho primário & NNT‡ \\
\hline \multicolumn{5}{|l|}{$\beta$-bloqueadores } \\
\hline CIBIS $\|^{9}$ & $\begin{array}{l}\text { Bisoprolol* } \\
10 \mathrm{mg} / \mathrm{d}\end{array}$ & $\begin{array}{c}2.647 \text { pacientes } \\
\text { NYHA III-IV } \\
\text { FEVE } \leq 35 \% \\
\text { Seguimento: } 16 \mathrm{~m} \\
\end{array}$ & $\begin{array}{c}\text { Mortalidade por todas as } \\
\text { causas } \\
\text { RRR }=34 \%\end{array}$ & 18 \\
\hline MERIT HF ${ }^{8}$ & $\begin{array}{l}\text { Succinato de metoprolol* } \\
\qquad 200 \mathrm{mg} / \mathrm{d}\end{array}$ & $\begin{array}{c}\text { 3.991 pacientes } \\
\text { NYHA II-IV } \\
\text { FEVE } \leq 40 \% \\
\text { Seguimento: } 12 \mathrm{~m}\end{array}$ & $\begin{array}{c}\text { Mortalidade por todas as } \\
\text { causas } \\
\text { RRR }=34 \%\end{array}$ & 27 \\
\hline COPERNICUS ${ }^{10}$ & $\begin{array}{l}\text { Carvedilol* } \\
25 \mathrm{mg} 2 \mathrm{xd}\end{array}$ & $\begin{array}{c}2.289 \text { pacientes } \\
\text { NYHA IV } \\
\text { FEVE }<25 \% \\
\text { Seguimento: } 11 \mathrm{~m}\end{array}$ & $\begin{array}{c}\text { Mortalidade por todas as } \\
\text { causas } \\
\text { RRR }=35 \%\end{array}$ & 15 \\
\hline \multicolumn{5}{|l|}{ IECAs/BRAS } \\
\hline SOLVD $^{2}$ & $\begin{array}{l}\text { Enalapril* } \\
10 \mathrm{mg} \mathrm{2xd}\end{array}$ & $\begin{array}{c}2.569 \text { pacientes } \\
\text { NYHA CF II-IV } \\
\text { FEVE } \leq 35 \% \\
\text { Seguimento: } 37 \mathrm{~m} \\
\end{array}$ & $\begin{array}{c}\text { Mortalidade de todas as causas } \\
\text { RRR }=16 \%\end{array}$ & 22 \\
\hline $\mathrm{CHARM}^{3}$ & $\begin{array}{l}\text { Candesartana* } \\
32 \mathrm{mg} / \mathrm{d}\end{array}$ & $\begin{array}{l}2.028 \text { pacientes } \\
\text { NYHA CF II-IV } \\
\text { FEVE < 40\% } \\
\text { Seguimento: } 37 \mathrm{~m} \\
\end{array}$ & $\begin{array}{c}\text { Mortalidade cardiovascular ou } \\
\text { HIC } \\
\text { RRR }=27 \%\end{array}$ & 14 \\
\hline \multicolumn{5}{|l|}{ AMR } \\
\hline RALES $^{4}$ & $\begin{array}{l}\text { Espironolactona* } \\
25-50 \mathrm{mg} / \mathrm{d}\end{array}$ & $\begin{array}{c}1.663 \text { pacientes } \\
\text { NYHA CF III-IV } \\
\text { FEVE } \leq 35 \% \\
\text { Seguimento: } 24 \mathrm{~m}\end{array}$ & $\begin{array}{c}\text { Mortalidade de todas as causas } \\
\text { RRR }=30 \%\end{array}$ & 10 \\
\hline EMPHASIS $^{5}$ & $\begin{array}{l}\text { Eplerenone }^{*} \\
25-50 \mathrm{mg} / \mathrm{d}\end{array}$ & $\begin{array}{l}2.737 \text { pacientes } \\
\text { NYHA CF II } \\
\text { FEVE } \leq 35 \% \\
\text { Seguimento: } 21 \mathrm{~m}\end{array}$ & $\begin{array}{c}\text { Mortalidade cardiovascular ou } \\
\text { HIC } \\
\text { RRR }=37 \%\end{array}$ & 13 \\
\hline \multicolumn{5}{|l|}{ INRA } \\
\hline PARADIGM-HF ${ }^{6}$ & $\begin{array}{l}\text { Sacubitril-Valsartana }{ }^{\dagger} \\
200 \mathrm{mg} 2 \mathrm{xd}\end{array}$ & $\begin{array}{c}8.442 \text { pacientes } \\
\text { NYHA CF II-IV } \\
\text { FEVE < 40\% / FEVE } \leq \\
35 \% \\
\text { Seguimento: } 27 \mathrm{~m} \\
\end{array}$ & $\begin{array}{c}\text { Mortalidade cardiovascular ou } \\
\text { HIC } \\
\text { RRR }=20 \%\end{array}$ & 21 \\
\hline \multicolumn{5}{|l|}{ Vasodilatadores diretos } \\
\hline A-HEFT 11 & $\begin{array}{c}\text { Hidralazina } \\
225 \mathrm{mg} / \mathrm{d} \\
+ \\
\text { Dinitrato de Isossorbida* } \\
120 \mathrm{mg} / \mathrm{d}\end{array}$ & $\begin{array}{c}1.050 \text { pacientes negros } \\
\text { NYHA III e IV } \\
\text { FEVE } \leq 35 \% \text {, ou FEVE < } \\
45 \% \text { se DDVE }>6.5 \mathrm{~cm} \text { ou } \\
>2,9 \mathrm{~cm} / \mathrm{m} 2 \\
\text { Seguimento: } 18 \mathrm{~m}\end{array}$ & $\begin{array}{l}\text { Morte por qualquer causa, } \\
\text { primeira HIC e mudança na } \\
\text { qualidade de vida } \\
\text { Mortalidade total } \\
\text { RRR }=43 \%\end{array}$ & 25 \\
\hline \multicolumn{5}{|l|}{ Inibidores If } \\
\hline SHIFT ${ }^{12}$ & $\begin{array}{c}\text { Ivabradina* } \\
5-7,5 \mathrm{mg} \mathrm{2xd}\end{array}$ & $\begin{array}{c}6.558 \text { pacientes } \\
\text { NYHA II - IV } \\
\text { FEVE < } 35 \% \\
\text { Ritmo sinusal / FC > 70 } \\
\text { Seguimento: } 23 \mathrm{~m} \\
\end{array}$ & $\begin{array}{c}\text { Morte cardiovascular ou HIC } \\
\text { RRR }=18 \%\end{array}$ & 26 \\
\hline \multicolumn{5}{|l|}{ Digitálicos } \\
\hline DIG $^{13}$ & $\begin{array}{l}\text { Digoxina* } \\
0,25 \mathrm{mg} / \mathrm{d}\end{array}$ & $\begin{array}{c}6.800 \text { pacientes } \\
\text { NYHA II - III } \\
\text { FEVE < } 45 \% \\
\text { Seguimento: } 37 \mathrm{~m} \\
\end{array}$ & $\begin{array}{l}\text { Mortalidade geral } \\
\text { Ausência de redução }\end{array}$ & NA \\
\hline \multicolumn{5}{|l|}{ Inibidores SGLT2 } \\
\hline DAPA-HF ${ }^{14}$ & $\begin{array}{l}\text { Dapagliflozina* } \\
10 \mathrm{mg} / \mathrm{dia}\end{array}$ & $\begin{array}{c}4.744 \text { pacientes } \\
\text { NYHA II-IV } \\
\text { FEVE < 40\% } \\
\text { Seguimento: } 18 \mathrm{~m} \\
\end{array}$ & $\begin{array}{c}\text { Mortalidade cardiovascular ou } \\
\text { HIC } \\
\text { RRR }=26 \%\end{array}$ & 21 \\
\hline EMPEROR-Reduced ${ }^{15}$ & Empagliflozina* 10 mg/dia & $\begin{array}{c}3.730 \text { pacientes } \\
\text { NYHA II-IV } \\
\text { FEVE < } 40 \% \\
\text { Seguimento: } 16 \mathrm{~m}\end{array}$ & $\begin{array}{c}\text { Mortalidade cardiovascular ou } \\
\text { HIC } \\
\text { RRR }=25 \%\end{array}$ & 19 \\
\hline \multicolumn{5}{|c|}{ Estimuladores da guanilato ciclase } \\
\hline VICTORIA ${ }^{16}$ & $\begin{array}{l}\text { Vericiguat }^{*} \\
10 \mathrm{mg} / \mathrm{d}\end{array}$ & $\begin{array}{c}5.050 \text { pacientes } \\
\text { NYHA II, III ou IV } \\
\text { FEVE < 45\% } \\
\text { Seguimento: } 11 \mathrm{~m}\end{array}$ & $\begin{array}{c}\text { Morte cardiovascular ou } \\
\text { primeira HIC } \\
\text { RRR }=10 \%\end{array}$ & 24 \\
\hline
\end{tabular}

*Versus Placebo. ${ }^{\dagger}$ Versus Enalapril. „NNT: definido para desfecho primário / morte por todas as causas no tempo total de seguimento. iECA: inibidores da enzima de conversão da angiotensina; BRA: bloqueadores dos receptores de angiotensina II; HIC: hospitalização por INRA: inibidores da neprilisina e bloqueadores dos receptores de angiotensina II; BB: betabloqueadores; AMR: antagonistas mineralocorticoides; NYHA: New York Heart Association; IC: insuficiência cardiaca; FEVE: fração de ejeção ventricular esquerda; iSGLT2: inibidores do cotransporte de sódio e glicose 2; FC: frequência cardíaca. 
um marcador de eventos em IC e um alvo terapêutico. Associase à redução do desfecho combinado de morte cardiovascular ou HIC em pacientes em ritmo sinusal, sintomáticos, com FC $\geq$ $70 b p m$ e fração de ejeção ventricular esquerda (FEVE) $\leq 35 \%$ ), sendo esse benefício basicamente por redução de HIC.

\section{Digoxina}

O uso de digoxina em pacientes com ICFEr foi avaliado na década de 90 e não se associou à redução de mortalidade em relação ao placebo. Houve, no entanto, redução das HIC..O efeito da digoxina e o seu lugar no tratamento contemporâneo da IC é desconhecido. Parece ser mais segura e efetiva na redução dos sintomas quando usada em baixas doses, guiada pelo nível plasmático e taxa de filtração glomerular(TFG).

Inovações no Tratamento Farmacológico

\section{Inibidores de SGLT2}

Os benefícios dos iSGLT2 na redução de eventos cardiovasculares adversos maiores e de $\mathrm{HIC}$ em pacientes com diabetes tipo 2 (DM2) foram observados inicialmente com a empagliflozina. Subsequentemente, diferentes iSGLT2 também demonstraram redução de HIC em pacientes diabéticos. Diante desses achados, os iSGLT2 foram avaliados em pacientes com IC.

No DAPA-HF (Dapagliflozin and Prevention of Adverse Outcomes in Heart Failure), 4.744 pacientes com ICFEr foram randomizados para receber dapagliflozina ou placebo além da terapia padrão, sendo 41,8\% com DM2. O desfecho primário de morte cardiovascular ou agravamento da IC foi significativamente menor no grupo dapagliflozina (26\% de redução). Quando analisados separadamente, houve redução significativa tanto na morte cardiovascular (18\% de redução) quanto na piora da IC (30\% de redução), independentemente de DM2. Tais resultados revelam uma nova terapia para $\mathrm{IC}$, já aprovada para essa finalidade.

O Empagliflozin Outcome Trial in Patients with Chronic Heart Failure and a Reduced Ejection Fraction (EMPEROR-Reduced) avaliou a empagliflozina versus placebo, além da terapia padrão, em 3.730 pacientes com ICFEr, sendo 50,2\% com DM2. Os pacientes apresentavam IC mais grave do que aqueles no DAPA$\mathrm{HF}$, com média de FEVE de $27 \%$ contra $31 \%$, sendo que mais de $70 \%$ dos pacientes tinham FEVE $<30 \%$, além de maior nível mediano de NT-proBNP (1.907 vs 1.437 pg/mL). Houve redução de $25 \%$ do desfecho primário de morte cardiovascular ou HIC a favor da empagliflozina. Quando analisados separadamente, não houve redução de morte cardiovascular, resultado diferente daquele observado no DAPA-HF. Novamente, o benefício foi visto independentemente da presença de DM2.

Esses dados confirmam os resultados do DAPA-HF e reforçam a justificativa para o uso de iSGLT2 em pacientes com ICFEr, para redução dos sintomas, melhora da qualidade de vida, redução do risco de hospitalização e morte cardiovascular.

\section{Estimuladores da Guanilato Ciclase Solúvel (GCs)}

Vericiguat, um novo estimulador da GCs, aumenta a produção de guanosina monofosfato (GMP) por estimulação direta da GCs, através de um sítio de ligação independente de óxido nítrico
(ON) e sensibiliza a GCs ao ON endógeno. Age suprindo o déficit relativo de produção de GMPc que ocorre na IC.

O ensaio clínico VICTORIA (Vericiguat in patients with reduced ejection fraction) alocou 5.050 pacientes com ICFEr, FEVE $<45 \%$, CF II-IV NYHA, para receber vericiguat, via oral, ou placebo, em adição à terapia padrão. O desfecho primário, morte cardiovascular ou primeira HIC ocorreu em 35,5\% versus 38,5\% dos pacientes em favor do vericiguat (NNT $=24$ em 11 meses). O benefício do desfecho composto deveu-se prioritariamente à redução de hospitalizações, não havendo benefício estatisticamente significativo em morte cardiovascular ou em mortalidade total.

Essa medicação tem potencial de integrar o grupo de medicações com efeito sobre sintomas e re-hospitalizações em pacientes com ICFEr, especialmente em pacientes com: hospitalizações frequentes, a despeito de terapia otimizada; pior função renal, já que o estudo considerava inclusão de pacientes com TFG > 15\%; ou com intolerância a outras drogas, sendo contraindicada em concomitância com nitratos.

Diante das novas evidências, dispomos de amplo arsenal terapêutico (Figura 1), capaz de impactar o prognóstico de pacientes com ICFEr. Uma vez iniciada terapia tripla, a otimização de doses e a individualização do tratamento, de acordo com o perfil do paciente, deve ocorrer precocemente, sabendo que há impacto em redução de mortalidade e HIC, quando acrescidas à terapia padrão.

Lista de Participantes do Heart Failure Summit Brazil 2020/ Departamento de Insuficiência Cardíaca - DEIC/SBC

Aguinaldo Freitas Junior, Andréia Biolo, Antonio Carlos Pereira Barretto, Antônio Lagoeiro Jorge, Bruno Biselli, Carlos Eduardo Montenegro, Denilson Campos de Albuquerque, Dirceu Rodrigues de Almeida, Edimar Alcides Bocchi, Edval Gomes dos Santos Júnior, Estêvão Lanna Figueiredo, Evandro Tinoco Mesquita, Fabiana G. Marcondes-Braga, Fábio Fernandes, Fabio Serra Silveira, Felix José Alvarez Ramires, Fernando Atik, Fernando Bacal, Flávio de Souza Brito, Germano Emilio Conceição Souza, Gustavo Calado de Aguiar Ribeiro, Humberto Villacorta Jr., Jefferson Luis Vieira , João David de Souza Neto, João Manoel Rossi Neto, José Albuquerque de Figueiredo Neto, Lídia Ana Zytynski Moura, Livia Adams Goldraich, Luís Beck-da-Silva Neto, Luís Eduardo Paim Rohde, Luiz Claudio Danzmann, Manoel Fernandes Canesin, Marcelo Bittencourt, Marcelo Westerlund Montera, Marcely Gimenes Bonatto, Marcus Vinicius Simões, Maria da Consolação Vieira Moreira, Miguel Morita Fernandes da Silva, Monica Samuel Avila, Mucio Tavares de Oliveira Junior, Nadine Clausell, Odilson Marcos Silvestre, Otavio Rizzi Coelho Filho, Pedro Vellosa Schwartzmann, Reinaldo Bulgarelli Bestetti, Ricardo Mourilhe Rocha, Sabrina Bernadez Pereira, Salvador Rassi, Sandrigo Mangini, Silvia Marinho Martins, Silvia Moreira Ayub Ferreira, Victor Sarli Issa.

\section{Contribuição dos autores}

Concepção e desenho da pesquisa, Obtenção de dados, Análise e interpretação dos dados, Redação do manuscrito e Revisão crítica do manuscrito quanto ao conteúdo intelectual importante: Marcondes-Braga FG, Ramires FJA, Figueiredo EL, Figueiredo Neto JA, Beck-da-Silva L, Rassi S. 


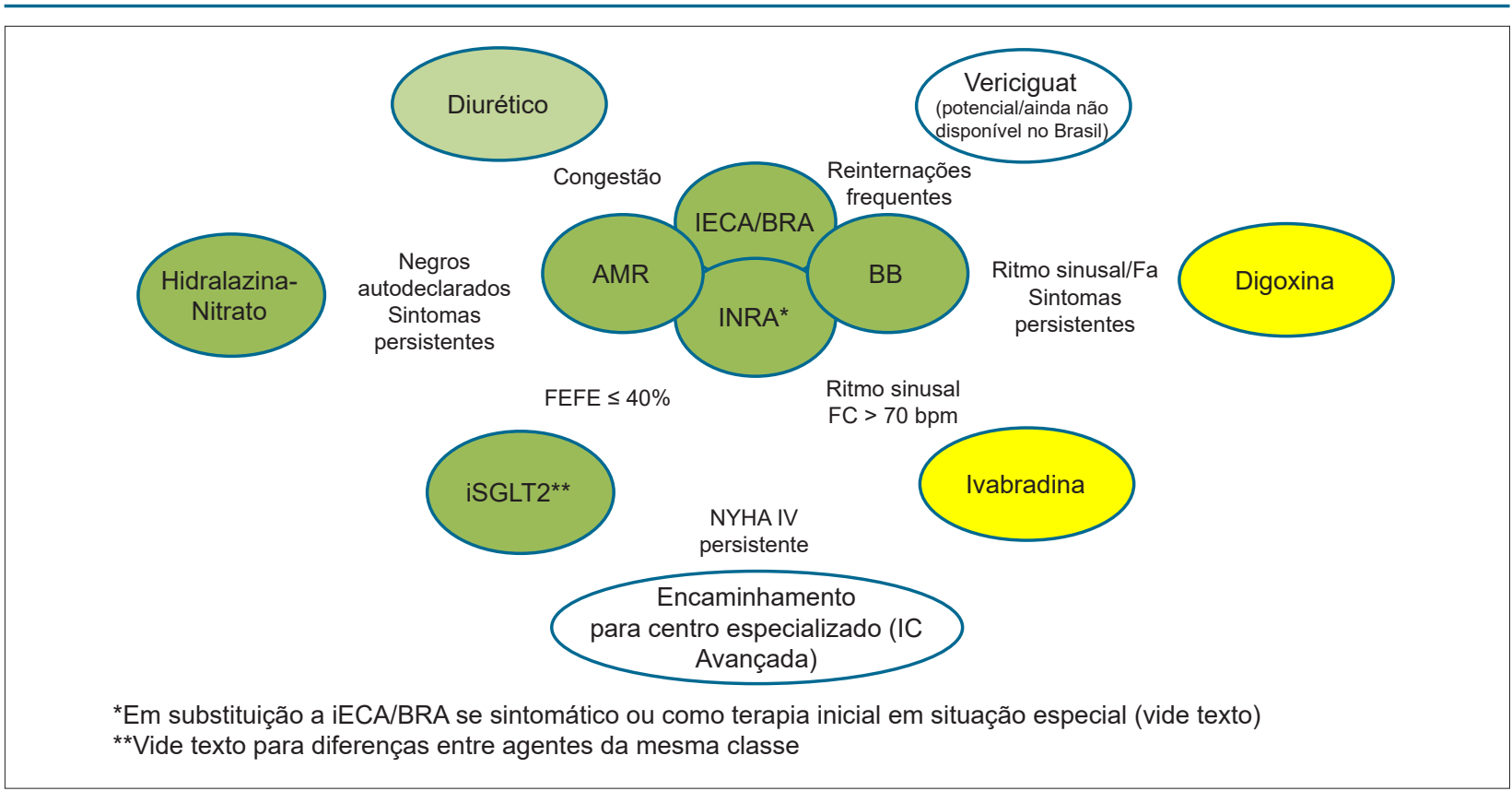

Figura 1-Abordagem farmacológica da Insuficiência cardiaca de fração de ejeção reduzida. iECA: inibidores da enzima de conversão da angiotensina; $B R A$ : bloqueadores dos receptores de angiotensina II; INRA: inibidores da neprilisina e bloqueadores dos receptores de angiotensina II; BB: betabloqueadores; AMR: antagonistas mineralocorticoides; NYHA: New York Heart Association; IC: insuficiência cardiaca; FEVE: fração de ejeção ventricular esquerda; iSGLT2: inibidores do cotransporte de sódio e glicose 2; FC: frequência cardiaca; FA: fibrilação atrial.

\section{Potencial conflito de interesses}

Fabiana G. Marcondes-Braga - recebe horonários por ministrar palestras e/ou fazer consultoria para os laboratórios Novartis, AstraZeneca e paticipei como subinvestigador em pesquisa clínica de Novartis e Amgen.

Felix J. A. Ramires - Recebi horonários por ministrar palestras, fazer consultoria e/ou pesquisa clínica para dos laboratórios Novartis, AstraZeneca, Amgen, Pfizer

Estêvão Lanna Figueiredo - Recebi horonários dos laboratórios Novartis, AstraZeneca, Boehringer e Bayer (palestras) e Novartis, AstraZeneca, Boehringer, Pfizer, Jansen, Bayer (pesquisa clínica)

José Albuquerque Figueiredo Neto - Recebi horonários dos laboratórios Novartis, AstraZeneca e Servier (palestras e consultoria) e Novartis (pesquisa clínica)

\section{Referências}

1. Comitê da diretriz brasileira de insuficiência cardíaca. Arq Bras Cardiol. 2018;111(3):436-539

2. SOLVD Investigators. Effect of enalapril on mortality and the development of heart failure in asymptomatic patients with reduced left ventricular ejection fractions. N Engl J Med 1992;327:685-91.

3. Granger GB, McMurray JJV, Yusuf S, Held P, Michelson EL, Olofsson B, et al. Effects of candesartan in patients with chronic heart failure and reduced left-ventricular systolic function intolerant to angiotensinconverting-enzyme inhibitors: the CHARM-Alternative trial. Lancet 2003;362:772-6.

4. Pitt B, Zannad F, Remme WJ, Cody R, Castaigne A, Perez A, et al. The effect of spironolactone on morbidity and mortality in patients with severe heart
Luís Beck-da-Silva - Recebi horonários dos laboratórios Novartis, AstraZeneca, Servier, Boehringer, Amgen (pesquisa clínica) e AstraZeneca, Novartis e Merck (palestras).

Salvador Rassi - Recebi horonários dos laboratórios Novartis, AstraZeneca, Servier, Boehringer, Amgen para palestras, consultoria e pesquisa clínica.

\section{Fontes de financiamento}

O presente estudo não teve fontes de financiamento externas.

\section{Vinculação acadêmica}

Não há vinculação deste estudo a programas de pósgraduação. failure. Randomized Aldactone Evaluation Study Investigators. N Engl J Med. 1999;341: 709-17

5. Zannad F, McMurray JJ, Krum H, van Veldhuisen DJ, Swedberg K, Shi H, et al. Eplerenone in patients with systolic heart failure and mild symptoms. N Engl J Med. 2011;364:11-21.

6. McMurray JJ, Packer M, Desai AS, Gong J, Lefkowitz MP, Rizkala AR, et al. Angiotensin-neprilysin inhibition versus enalapril in heart failure. N Engl J Med. 2014 Sep 11;371:993-1004.

7. Velazquez EJ, Morrow DA, DeVore AD, Duffy Cl, Ambrosy AP, McCague $\mathrm{K}$, et al. Angiotensin-Neprilysin Inhibition in Acute Decompensated Heart Failure. N Engl J Med.2019;380:539-548. 
8. Taylor AL, Ziesche S, Yancy C, Carson P, D'Agostino JrR, Ferdinand K.et al.for the African-American Heart Failure Trial Investigators. Combination of isosorbide dinitrate and hydralazine in blacks with heart failure. N Engl J Med. 2004; 351: 2049-2057.

9. Swedberg K, Komajda M, Böhm M, Borer JS, Ford I, Dubost-Brama A, et al. SHIFT Investigators. Ivabradine and outcomes in chronic heart failure (SHIFT): a randomised placebo-controlled study. Lancet. 2010Sep 11;376(9744):875-85.

10. igitalis Investigation Group. The effect of digoxin on mortality and morbidity in patients with heart failure. N Engl J Med. 1997;336(8):525-533.
11. McMurray JJV, Solomon SD, Inzucchi SE, Køber L, Kosiborod MN, Martinez FA, et al. Dapagliflozinin patients with heart failure and reduced ejection fraction. N EnglJ Med 2019; 381: 1995-2008.

12. Packer M, Anker SD, Butler J, Filippatos G, Pocock SJ, Carson P, et al Cardiovascular and renalo utcomes with empagliflozin in heart failure. $\mathrm{N}$ Engl J Med. DOI: 10.1056/NEJMoa2022190.

13. Armstrong PW, Pieske B, Anstrom KJ, Ezekowitz J, Hernandez AF, Butler J, et al. Vericiguat in Patients with Heart Failure and Reduced Ejection Fraction. N Engl J Med 2020;382:1883-93. 